\title{
Predicting Discharge Outcomes of VA Nursing Home Residents
}

\author{
DAVID R. MEHR, MD, MS \\ University of Missouri-Columbia \\ BRENT C. WILLIAMS, MD, MPH \\ University of Michigan Medical Center \\ BRANT E. FRIES, PhD \\ University of Michigan and Ann Arbor VA Medical Center
}

This article's purpose was to identify predictors of discharge outcomes of VAnursing home stays. Using data tapes, diagnostic and assessment data were assembled on elderly individuals admitted to VA nursing homes nationwide during Fiscal Year 1987. Six-month outcomes for 3 groups were considered: all residents $(n=5,895)$, and those remaining in care after $6(n=2,815)$ and 12 months $(n=1,812)$, respectively. Logistic regression was used to evaluate predictors of death and community discharge. Limited activities of daily living (ADL) dependency, younger age, and receipt of rehabilitation services most consistently predicted community discharge. ADL dependency, older age, oxygen use, terminally ill prognosis, malignancy, and congestive heart failure most consistently predicted mortality. For both dependent variables, predictive ability declined as stay length increased. Predicting death and community discharge become increasingly problematic as stay lengthens. Comparing observed versus expected discharge outcomes has limited usefulness as a quality-improvement tool.

For more than a decade, Robert Kane has advocated using observed versus expected outcomes of nursing home care as a measure of quality and a criterion for reimbursement (Kane, 1990, 1995; Kane, Bell, Riegler, Wilson, \& Keeler, 1983). Such a system has the intuitive

AUTHORS' NOTE: This article was supported in part by a pilot feasibility grant from the Claude D. Pepper Geriatric Research and Training Center at the University of Michigan (National Institute of Aging grant AG-08808) and grant IIR 90-096 from the Department of Veterans Affairs. A preliminary version of this article was presented at the 44th Annual Meeting of The

JOURNAL OF AGING AND HEALTH, Vol. 9 No. 2, May 1997 244-265

(c) 1997 Sage Publications, Inc. 
appeal of creating incentives for better care; however, it demands effective outcome prediction. In their original work, Kane and colleagues were relatively successful in estimating future values of several numerically scaled variables, such as activities of daily living (ADL) status. However, death and community discharge eluded predictability. We wondered whether these outcomes were inherently unpredictable or whether sampling and data limitations had handicapped previous studies.

Several studies using multivariable methods have examined discharge outcomes of nursing home residents (Engle \& Graney, 1993; Greene \& Ondrich, 1990; Kane et al., 1983; Kiel, Eichorn, Intrator, Silliman, \& Mor, 1994; Lewis, Kane, Cretin, \& Clark, 1985; Lewis, Leake, Clark, \& Leal-Sotelo, 1989; Retsinas \& Garrity, 1986; Weissert \& Scanlon, 1985). These studies are heterogeneous in design, population, and analytic method, though most used admission (Engle \& Graney, 1993; Kiel et al., 1994; Lewis et al., 1989) or discharge cohorts (Lewis, Kane et al., 1985; Retsinas \& Garrity, 1986; Weissert \& Scanlon, 1985). The majority considered acute-care hospitalization as a final outcome (Kane et al., 1983; Kiel et al., 1994; Lewis, Kane et al., 1985; Lewis et al., 1989; Weissert \& Scanlon, 1985). Two studies treated hospitalization as an intermediary outcome and followed residents to community discharge, death, or transfer to another nursing home (Engle \& Graney, 1993; Retsinas \& Garrity, 1986).

Definitions of potential predictor variables also were heterogeneous, which limits comparisons. Although older age frequently predicted death or lower probability of community discharge (Engle \& Graney, 1993; Greene \& Ondrich, 1990; Kane et al., 1983; Kiel et al., 1994; Lewis et al., 1989; Weissert \& Scanlon, 1985), no other sociodemographic variable consistently related to these outcomes. Measures of functional impairment-represented by varying combinations of impaired ADL, cognition, continence, and ambulation-were among

Gerontological Society of America, November 1991. Elizabeth Bates and her staff at the Great Lakes Health Services Research and Development Office of the Department of Veterans Affairs provided access to and assistance with the data. Chris Young, Anna Davidson, and Charles Betley assisted with programming and data analysis. Andrzej Galecki provided statistical assistance. Robin Kruse prepared the figures and Karen Davenport assisted with manuscript preparation. Address requests for reprints and correspondence to David R. Mehr, MD, MS, M228 Medical Sciences, Department of Family and Community Medicine, University of Missouri-Columbia, Columbia, MO 65212. 
the most important outcome predictors. With rare exceptions, they lowered the probability of community discharge and raised the probability of death (Engle \& Graney, 1993; Greene \& Ondrich, 1990; Kiel et al., 1994; Lewis, Kane et al., 1985; Lewis et al., 1989; Weissert \& Scanlon, 1985). Several economic variables, such as source of nursing home payment or facility Medicaid percentage, were statistically significant in one or more studies, but the relationships were not consistent (Engle \& Graney, 1993; Lewis, Kane et al., 1985; Weissert \& Scanlon, 1985).

Five studies reported specific diagnostic data. Malignancy was strongly associated with death in two studies (Lewis et al., 1989; Engle \& Graney, 1993). Several diagnoses were associated with decreased probability of community discharge: malignancy (Weissert \& Scanlon, 1985); dementia, neurologic disorder, or mental disorder (Engle \& Graney, 1993; Kiel et al., 1994; Weissert \& Scanlon, 1985); and stroke (Weissert \& Scanlon, 1985). Fracture or hip fracture were positively associated with community discharge in three studies (Lewis, Kane et al., 1985; Engle \& Graney, 1993; Weissert \& Scanlon, 1985).

In considering nursing home outcomes, two special methodological problems deserve comment. First, sampling is problematic because nursing homes contain a mix of populations: a larger long-stay group with a low discharge rate and a smaller short-stay group with high turnover (Keeler, Kane, \& Solomon, 1981). As a result, cross-sectional or prevalence sampling predominantly includes long-stay residents. Discharge sampling predominantly includes short-stay residents but provides a mix between admission and cross-sectional samples (Wayne, Rhyne, Thompson, \& Davis, 1991). Second, nursing home discharge data are misleading because many such moves are to hospitals or to other nursing facilities (Hing, Sekscenski, \& Strahan, 1989; Lewis, Cretin, \& Kane, 1985). Because most nursing home residents admitted to an acute-care hospital either die or return to a nursing home (Hing et al., 1989; Narain et al., 1988), hospitalization is an intermediate rather than a final outcome. Therefore, samples based on all nursing home discharges will substantially underestimate length of stay and the proportion of residents ultimately dying at the conclusion of a nursing home stay (Lewis, Cretin et al., 1985; Spence \& Wiener, 1990). 
In this article, we report our efforts to explore in more detail the problem of predicting the ultimate discharge outcomes of nursing home residents. We hoped that separating residents into groups of different stay length, looking at ultimate care outcomes, and using a very large data set with functional as well as diagnostic data would lead to better predictors of discharge status. Better prediction could facilitate reimbursement and quality improvement methodologies based on resident outcomes.

\section{Method}

\section{DATA SOURCES}

Our subjects were residents of Department of Veterans Affairs (VA) nursing homes. The VA routinely collects patient information in two national data files. Each annual Patient Treatment File (PTF) records all discharges during that fiscal year from any VA-supported institutional care, including VA hospitals, VA nursing home care units, and contract community nursing homes. Patient records include data on demographics, admission, discharge, procedures, and discharge diagnoses. The Patient Assessment File (PAF) details admission and semiannual assessments for each nursing home resident. These assessments enable resident classification according to the Resource Utilization Groups, Version 2 (RUG-II) system (Schneider, Fries, Foley, Desmond, \& Gormley, 1988). As the PTF includes hospital discharges for community dwellers, many more individuals are represented in the PTF than the PAF.

\section{STUDY POPULATION}

We studied all elderly residents (65 years of age or older) newly admitted to VA nursing homes nationwide during Fiscal Year (FY) 1987 (October 1, 1986, to September 30, 1987). To identify these individuals, we completed the following five steps: (a) We searched PAF files to identify all those having a nursing home assessment in FY 1987 ( $n=23,029)$; (b) using social security number as a common identifier, we located and combined all PAF, PTF, and National Death 
Index records for these individuals into a master database; (c) we eliminated individuals with seriously flawed records; (d) we dropped those younger than 65 years of age or not newly admitted; and (e) we selected three cohorts to analyze stay outcomes. These steps are described in more detail in the following.

Records abstracted for the master database included (a) all PAF nursing home assessments from FY 1987 to FY 1989 (October 1986 to October 1989), (b) all PTF discharges from FY 1986 to FY 1989 (October 1985 to October 1989), and (c) deaths recorded in the National Death Index through October 1989. We incorporated each individual's data from these sources into a single longitudinal record of hospitalizations, nursing home stays, nursing home assessments, and death if it occurred. We chose FY 1987 for identifying nursing home residents to ensure that facilities had collected PAF data for at least 1 year. The FY 1986 PTF information enabled us to identify the beginning of nursing home stays and to assess hospital and nursing home use prior to nursing home entry in FY 1987. Further details concerning this master database have been reported previously (Williams, Mehr, \& Fries, 1994).

We excluded 1,971 individuals $(8.6 \%)$ whose records were seriously flawed. Of the entire data set, $6.7 \%$ of the residents had records with seriously conflicting information, such as being assessed in a nursing home during the middle of a hospital stay. An additional 1.9\% had no information except a single assessment during the entire 4-year period.

We then selected all residents over the age of 65 years with a new admission to a VA nursing home care unit after September 15, 1986 $(n=6,467)$. We term this the "index" admission to distinguish it from prior nursing home stays. We considered nursing home stays to be terminated only by death or discharge from all institutional care for at least 1 week. Hospitalization from a nursing home, followed by the individual returning to a nursing home, was considered the same nursing home stay. Contract community nursing homes were not distinguished from VA nursing homes in this regard, except that only VA nursing home admissions could constitute the index admission.

In our analyses, we considered 6-month outcomes for three cohorts of elderly nursing home residents: (a) all newly admitted residents (the all-admissions cohort), (b) residents remaining in institutional care 6 


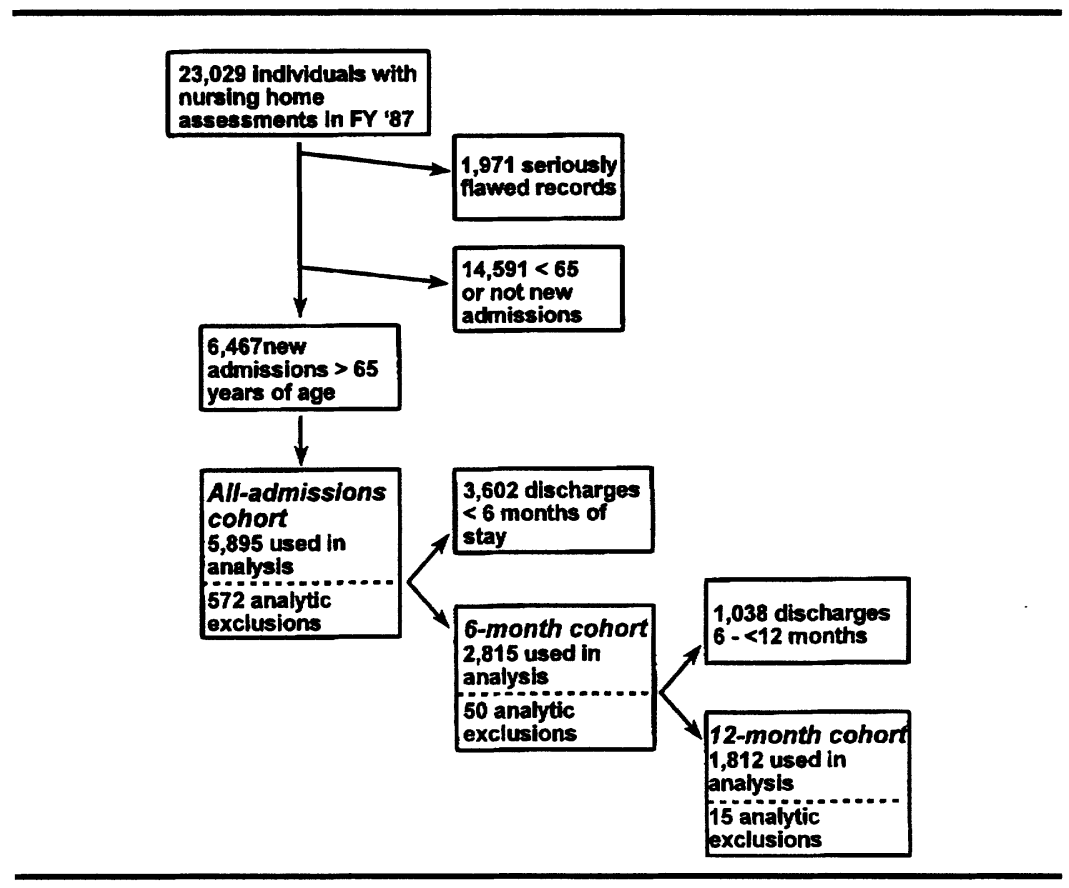

Figure 1. Identification of the three study cohorts.

months after the index admission (the 6-month cohort), and (c) residents remaining in institutional care 12 months after the index admission (the 12-month cohort). To investigate each cohort's outcomes, we made some analytic exclusions. For the all-admissions cohort, we excluded 492 individuals (7.6\%) who had no assessment within 30 days of nursing home entry and $80(1.2 \%)$ additional individuals who had incomplete records terminating during the first 6 months of stay. Similarly, the small number of analytic exclusions for the 6- and 12-month cohorts either (a) lacked an assessment before 6 or 12 months of stay, respectively, or (b) had an incomplete record terminating during that cohort's outcome period. Individuals lacking a timely assessment were eligible for inclusion in subsequent cohorts. Figure 1 shows the process for identifying the three study cohorts. After ana1ytic exclusions, there were 5,895 individuals in the all-admissions cohort, 2,815 in the 6-month cohort, and 1,812 in the 12-month cohort. 


\section{STAY OUTCOMES}

We examined the probability of death or community discharge terminating a nursing home stay over the succeeding 6 months for each of the three length-of-stay cohorts. Death was identified from VA discharge records or the National Death Index. There was a very high degree of agreement between the two sources (Williams, Demitrack, \& Fries, 1992). We defined community discharge as discharge out of VA institutional care for at least 1 week with no indication that the discharge destination was another hospital or nursing home. We chose the term "community discharge" rather than "live discharge" to emphasize our exclusion of transfer to another institution. Asmall number of residents were transferred to a nursing home completely outside the VA system or remained in a contract community nursing home at the contract's expiration. We counted such residents as remaining in nursing home care at the end of that length-of-stay cohort's 6-month outcome period; however, we treated them as discharged - that is, not eligible to be future subjects-when identifying subsequent cohorts.

\section{ANALYSIS}

We decided to examine three distinct length-of-stay cohorts for two reasons: (a) the known differences between short-stay and long-stay residents; and (b) the failure of proportional hazards models to meet the key proportionality assumption. In evaluating potential predictor variables for the three cohorts, we used the most recent information up to nursing home entry, 6 , and 12 months of stay, respectively. For the all-admissions cohort, the first nursing home assessment was also included.

We considered potential predictors from the following categories: sociodemographic, functional (ADLlimitations), conditions and treatments, medical diagnoses, and health services use. Sociodemographic variables included age at nursing home admission (65-74, 75-84, and $85+$ years of age), gender, marital status, and ethnic and racial status (White non-Hispanic vs. others). The limited income information available was not useful because almost all veterans were in the lowest income classification. 
Measures of function, conditions, and treatments were derived from nursing home assessments. We measured ADL limitations with a four-level scale ranging from minimal or no dependency to highly dependent. The scale was based on the RUG-II ADL index (Schneider et al., 1988), which sums individual dependency scores for toileting (range 1-3), transferring (range 1-3), and eating (range 1-4) to create an overall ADL dependency measure. In each case, 1 represents independent or needing at most minimal assistance. The highest individual dependency scores represent the following: for toileting, "incontinent-taken to the toilet on a regular basis"; for transferring, "continuous physical assistance of two persons or bedfast"; and for eating, "tube or parenteral feeding." We derived the scale for this study by collapsing the RUG-II ADL index into four groups: 3,4 to 5, 6 to 7 , and 8 to 10 . Conditions and treatments refer to the 4 weeks preceding an assessment and included heavy rehabilitation programs (services at least 5 days per week), severe behavior problems, assessment as terminally ill, current oxygen use, recent urinary tract infection, and recent episode of dehydration.

Concerning health services use, we considered prior hospital or nursing home stays and hospitalization from the nursing home. Prior stays were defined as care under VA auspices before the index nursing home admission. However, because discharge data went back only to October 1985, each individual's records concerning prior stays cover only 1 to 2 years. Hospitalization from the nursing home refers to hospital stays after the index admission. For the 6- and 12-month cohorts, we considered hospitalizations that occurred before 6 and 12 months of stay, respectively.

Finally, we considered diagnoses that were either prevalent in the data set or deemed important by the two physician authors. We derived the following diagnostic variables from grouped ICD-9-CM (international classification of diseases, 9th edition, in clinical modification) codes: alcoholism, anemia, arthritis, atherosclerotic heart disease, cerebrovascular disease, chronic obstructive pulmonary disease (COPD), congestive heart failure, dementia (including degenerative brain disorders), diabetes mellitus, fluid or electrolyte disorder, hip fracture, hypertension, infections other than pneumonia, kidney disease, malignancy, pneumonia, and major psychiatric disorders. We 
used all listed diagnoses; up to 10 were provided for each hospital or nursing home discharge. For the all-admissions cohort, we used diagnoses from before entry into the cohort. For the 6-month and 12-month cohorts, we also added diagnoses from hospitalizations from the nursing home up to 6 and 12 months, respectively. For each analysis, some individuals had no diagnostic data because they had no hospital or nursing home discharge up to that time of stay; in this situation, we considered diagnostic information missing. Because hip fractures occurred very rarely after nursing home admission, we considered them only for the all-admissions cohort.

Using SAS statistical software (1992), variables were first evaluated with individual chi-square tests of proportions and then in multivariable logistic regression. With two exceptions, each chi-square test is based on a $3 \times 2$ table of the three possible outcomes (remaining in the nursing home, being discharged to the community, or dying) with a variable being present or absent. The tables for age groups and ADL dependency include the three and four levels, respectively, for these variables. The null hypothesis for each variable is that the proportion of residents remaining in the nursing home, being discharged to the community, or dying is the same for each value of the variable.

For the logistic regressions, we separately examined two dependent variables: community discharge and death. This does not treat them as competing risks, as would be possible with multinomial logistic regression or proportional hazards regression. Nonetheless, our approach provides highly interpretable estimates of the probability of these two states individually given specific patient characteristics.

Because our primary purpose was finding the best predictive models, we used stepwise regressions to select the most important variables in three stages. We set the significance criterion for inclusion in a model at alpha $=.05$. All models explicitly included age and ADL status. We grouped age and ADL data in logical categories and confirmed that in each case this produced an approximately linear relation with the logarithm of the odds for death and community discharge (Hosmer \& Lemeshow, 1989). Because some individuals had no diagnostic data, we first evaluated variables other than diagnoses and then tested diagnostic variables. Finally, we tested interaction terms for age and ADL dependency with all other variables in the 
models. From coefficients and standard errors of the final models, we calculated adjusted odds ratios and confidence intervals. In the one instance of a significant interaction term-age by malignancy in the 6-month cohort-we report the adjusted odds ratio for the middle (75-84 years old) age group.

Initial modeling for the all-admissions cohort was done on a $50 \%$ systematic sample obtained by selecting every other record ordered by social security number. Subsequently, we refined our best model by comparison with the validation sample and dropped variables and interaction terms not significant in both subsets. The final model revealed relatively stable coefficients in both subsets. We report results for the validation sample. We did not carry out split-sample modeling in the 6- and 12-month cohorts because the reduced sample sizes led to few variables being significant. Therefore, for these two cohorts we derived the best fitting models for the entire sample and report these results.

Results

Figure 2 shows the overall 6-month outcomes for the three cohorts. Community discharge is quite common during the first 6 months after nursing home admission (38.6\% of the all-admissions cohort). Residents who remain beyond 6 months are increasingly likely to persist in care. Six-month probability of death declines slightly from $17.7 \%$ for the all-admissions cohort to $12.8 \%$ for the 12-month cohort. Table 1 shows the proportion of individuals in each cohort with a particular characteristic who died or were discharged to the community over the next 6 months. Because of the large sample sizes, most variables are statistically significant even with relatively small differences from the overall figure.

In multivariable analysis with community discharge as the dependent variable (Table 2), age, ADL limitations, and participation in an active rehabilitation program are the only three predictors consistent across the cohorts. However, as measured by odds-ratio magnitudes, the strength of the association varies. Of note, participation in a heavy rehabilitation program is a particularly strong predictor of community discharge for the 6-month cohort. The only four significant diagnoses 


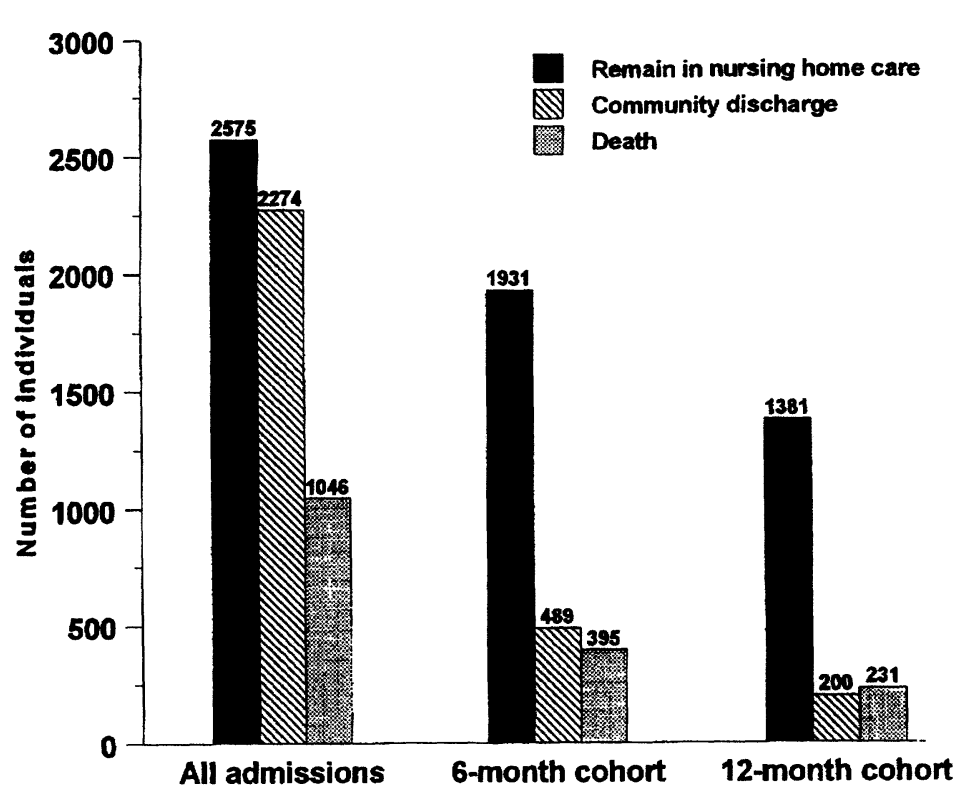

Figure 2. Six-month outcomes of VA nursing home residents by length-of-stay cohorts.

all predict a lower probability of community discharge, and diagnoses clearly become less important as length of stay increases.

In contrast, with death as the dependent variable (Table 3), diagnoses are prominent as predictors, particularly in the 6-month cohort. Significant ADL limitation is a very strong predictor of mortality in the all-admissions cohort, but no more important than age for the other two cohorts. Similar to the univariate results, other consistent predictors include oxygen use, terminally ill prognosis, congestive heart failure, and malignancy. An age-by-malignancy interaction was found in the 6-month cohort only. For this cohort, malignancy is less predictive of mortality at older ages. Being hospitalized from the nursing home predicts increased mortality in both the 6- and 12-month cohorts (not applicable in the all-admissions cohort); however, it also predicts community discharge in the 12-month cohort. 


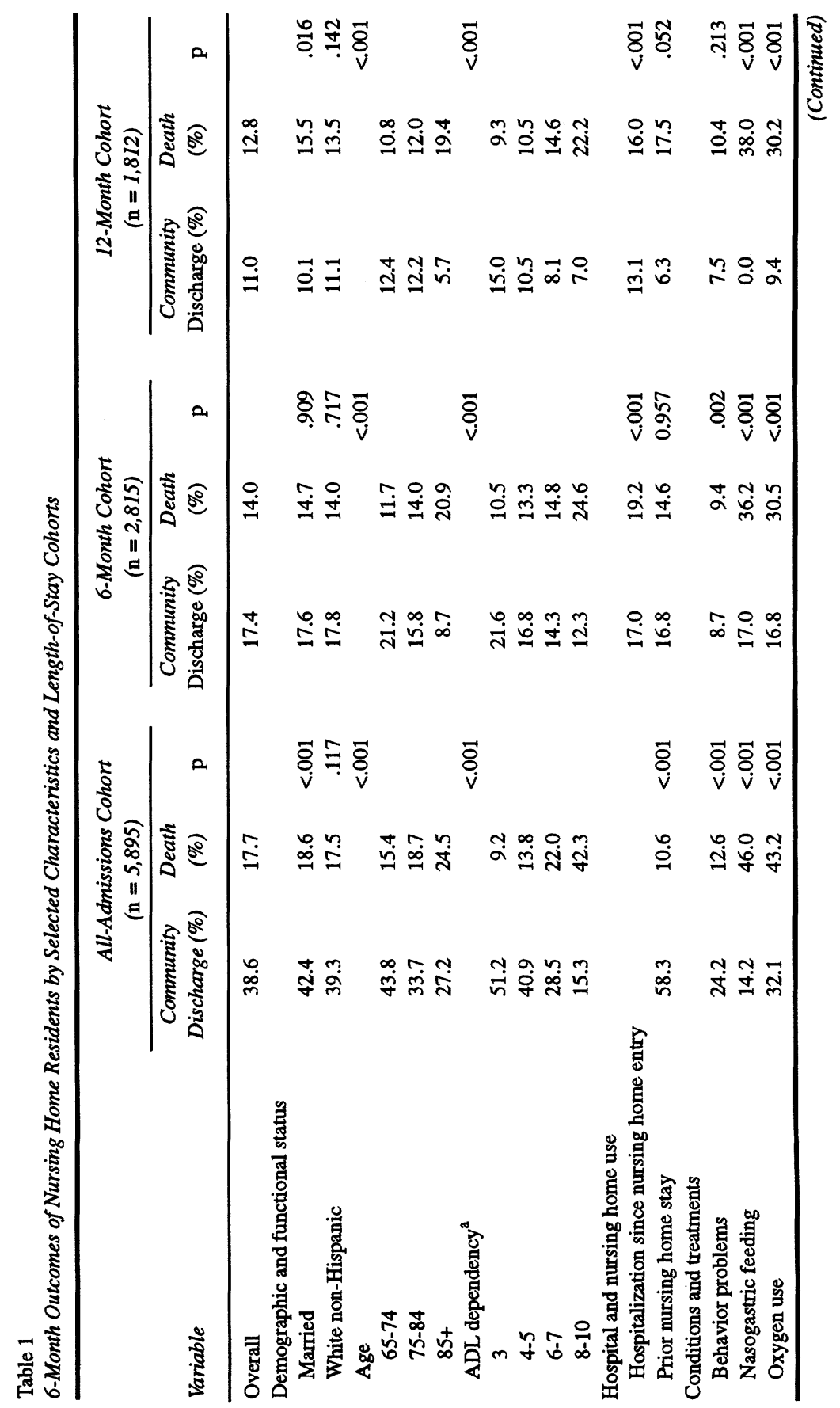




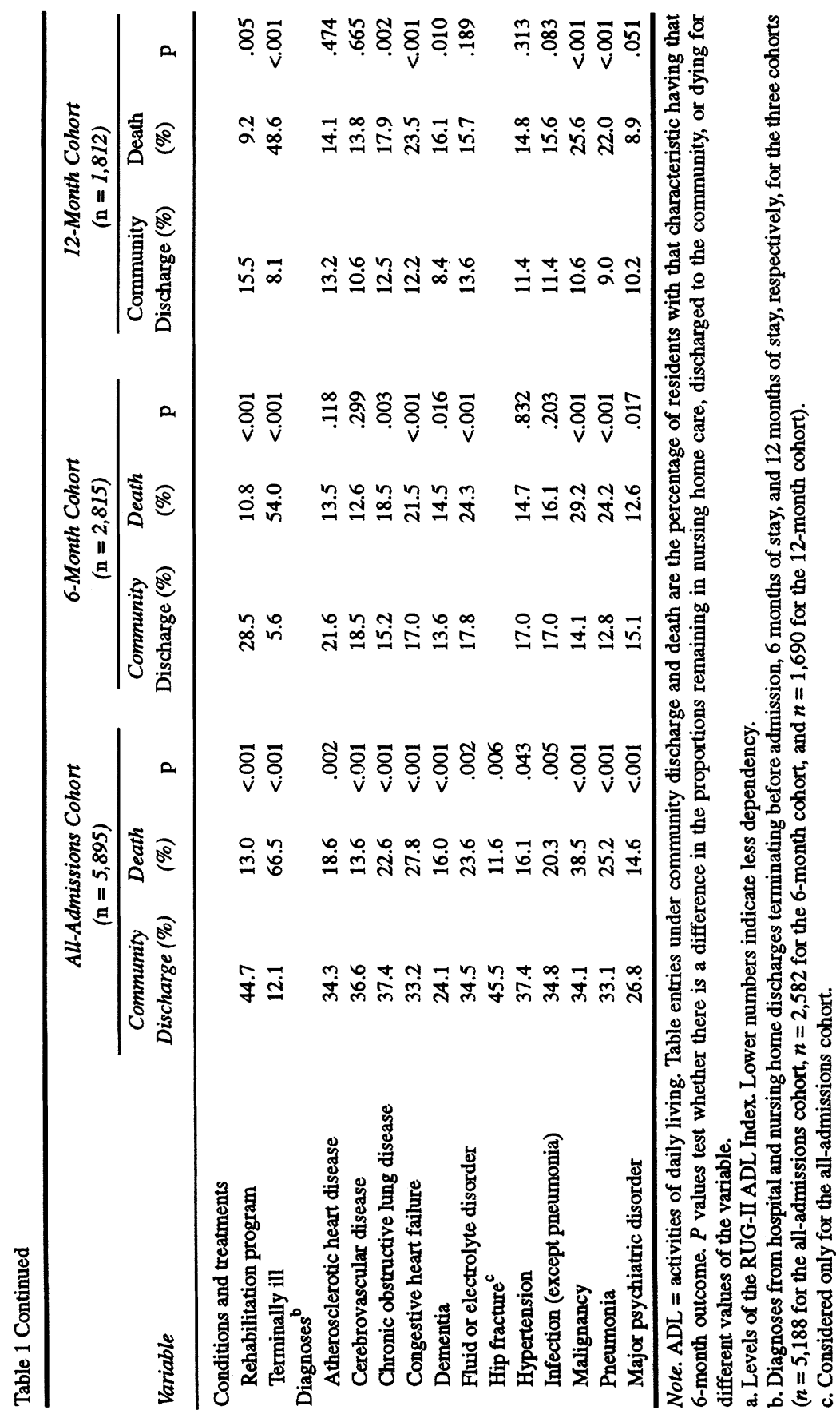




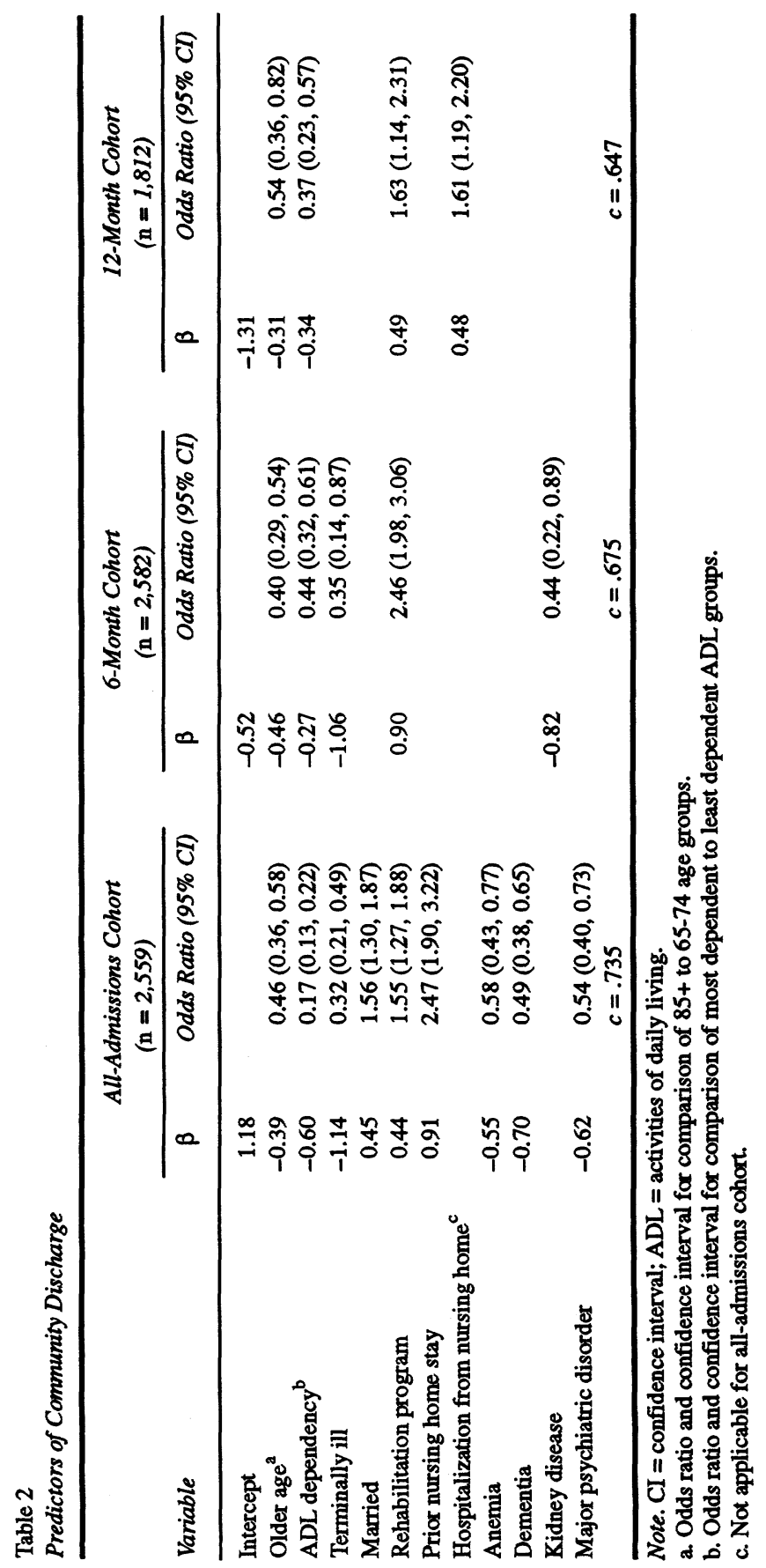




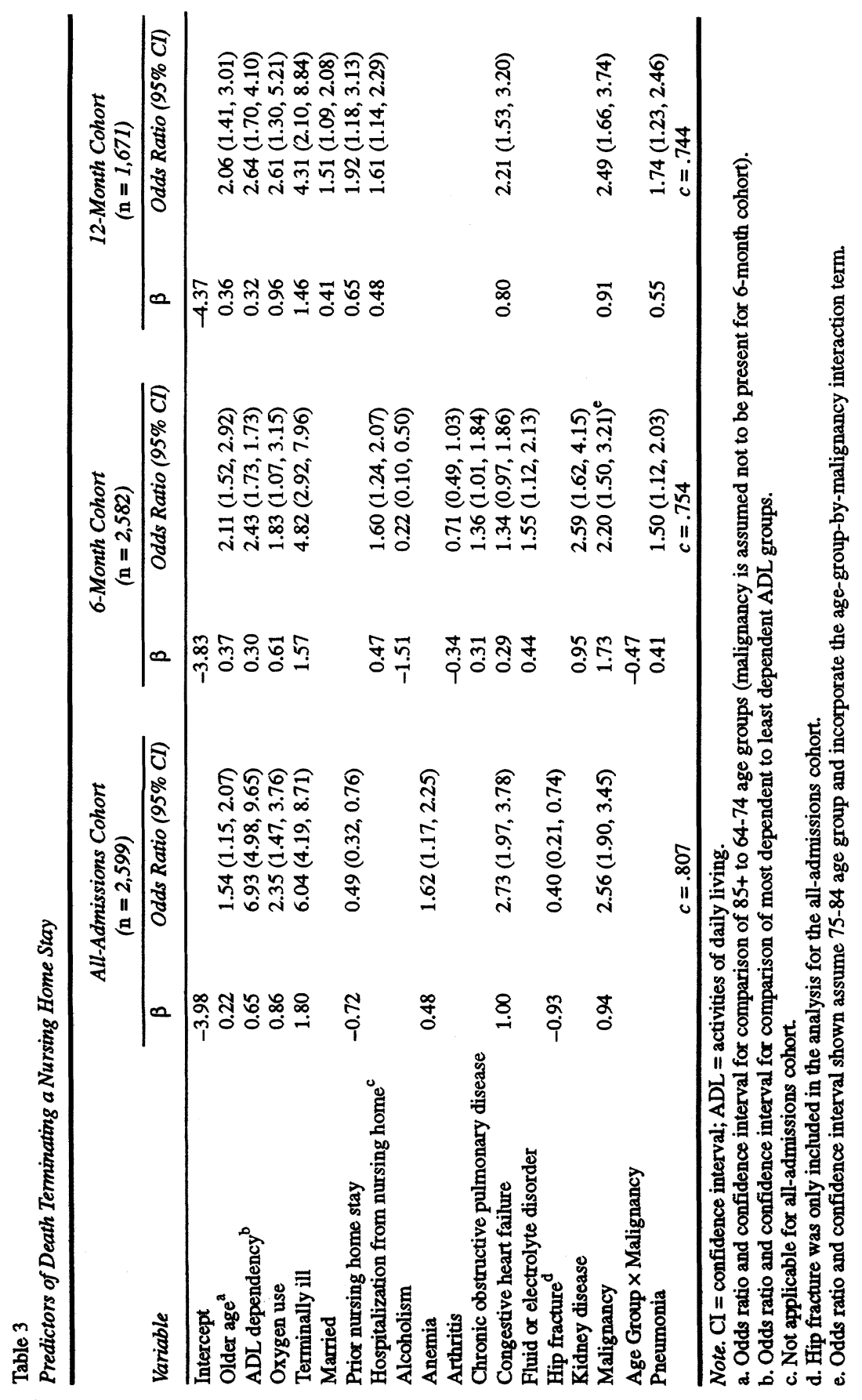


Based on the $c$ statistic-equal to the area under the receiver operating characteristic (ROC) curve-models predicting community discharge only modestly discriminate for the all-admissions cohort and less so for the two subsequent length-of-stay cohorts. Ability to predict death also declines with increasing length of nursing home stay, though discrimination is better than for community discharge.

\section{Discussion}

We found that models predicting community discharge from VA nursing homes had only modest predictive ability. Models predicting death performed somewhat better. But for both outcomes, the predictive ability declined as the length of stay increased. Further, the importance of individual variables as predictors changed markedly across our length-of-stay cohorts. These changes are not surprising given previous work demonstrating differences between short- and long-stay nursing home populations (Keeler et al., 1981).

Contrary to our expectations, we did not find that taking this and other methodological issues into account led to useful predictive models for outcomes-based quality assessment. Perhaps such models could be helpful with newly admitted residents, but the vast majority of residents are long stayers. Further, although predicting death in this population worked better than predicting community discharge, death is a particularly problematic outcome for assessing quality of care. For some nursing home residents, death is an appropriate outcome. Our findings support the recommendation that outcomes other than discharge status be the primary measures for assessing quality.

Turning to specific variables, we found that ADL limitations, age, terminal illness, and most diagnoses behaved as expected. Participating in a heavy rehabilitation program consistently predicted community discharge. Surprisingly this was most strongly associated with community discharge in the 6-month cohort. This may reflect the availability of unusually long-term rehabilitation, which we have observed in VA nursing home units. Or it could reflect a slower pace of care.

In community nursing home settings, medium ADL dependency has been associated with receipt of rehabilitation services (Murtaugh, 
Cooney, DerSimonian, Smits, \& Fetter, 1988). Nonetheless, we did not find an interaction between ADL dependency and heavy rehabilitation in our logistic regression modeling. This means that for our subjects, the influence of ADL limitations on community discharge was not affected by receiving rehabilitation, but rehabilitation independently increased the probability of community discharge. Other reports on the outcomes of nursing home care have not explicitly considered receipt of rehabilitation services, though Kiel et al. (1994) noted in their report of hip fracture patients that virtually all were receiving physical therapy.

Our logistic regression findings concerning marital status are more surprising. Being married is associated with a higher probability of community discharge only in the all-admissions cohort. It is associated with a higher probability of mortality in the 12-month cohort. One previous study found marital status associated with higher probability of community discharge (Weissert \& Scanlon, 1985), and another found being married associated with death as a 6-month discharge outcome (Engle \& Graney, 1993). The presence of supports and assistance in the community would logically enhance the probability of early nursing home discharge, though this effect might wane with time. Perhaps remaining in a nursing home while married indicates increased illness severity.

Hospital use offers some similarly puzzling patterns. Hospitalization after nursing home entry predicted a higher probability of death in the 6-month and 12-month cohorts. But it also predicted a higher probability of community discharge in the 12-month cohort. Perhaps a subgroup of longer-stay individuals require acute-care interventions to enable community discharge.

We also found that a prior nursing home stay predisposed an individual to increased probability of community discharge and lower probability of death in the all-admissions cohort but a higher probability of death in the 12-month cohort. These residents with prior nursing home stays tend to have repeated very short nursing home stays and may be unique in the VA (Williams, Fries, \& Mehr, 1993, 1996). However, most other studies have not specifically examined this group. Lewis and colleagues (1989) did report that readmission 
from home was associated with a substantially lower probability of community discharge than readmission from the hospital.

Our findings concerning diagnostic variables are generally in expected directions and consistent with previous studies, though we are the first to report the substantial variation with length of stay in the predictive significance of most diagnoses. As have others (Engle \& Graney, 1993; Lewis et al., 1989), we found malignancy to be consistently associated with increased probability of mortality in all periods. This occurred despite our simultaneously controlling for terminally ill prognosis in the model. Congestive heart failure in all periods and pneumonia in the 6- and 12-month cohorts were the other diagnostic variables most consistently associated with mortality. Neither of these is surprising. COPD is only significantly associated with mortality in the 6-month cohort. Perhaps oxygen use, potentially a measure of disease severity, predicts mortality better than a COPD diagnosis in this population.

We found dementia and major psychiatric disorders associated with decreased probability of community discharge in the all-admissions cohort. This is consistent with previous work (Engle \& Graney, 1993; Kiel et al., 1994; Weissert \& Scanlon, 1985). We did not find the previously reported association between hip fracture and increased probability of community discharge (Engle \& Graney, 1993; Lewis, Kane et al., 1985; Weissert \& Scanlon, 1985), but hip fracture was associated with a lower probability of mortality in the all-admissions cohort. Perhaps heavy rehabilitation is a better marker for successful recovery from hip fracture.

Diagnoses in general were far more useful in predicting death than community discharge, and most diagnoses were predictive of a higher probability of mortality. The only variables, other than hip fracture, associated with a lower probability of mortality were alcoholism and arthritis (both only in the 6-month cohort). These two variables did not have any association, positive or negative, with community discharge. The strength of the alcoholism relationship (odds ratio 0.22 , $95 \%$ confidence interval 0.10 to 0.50 ) is impressive. These chronic conditions might predispose to persistence in nursing home care, but it is puzzling that they are only significant for residents remaining in institutional care for 6 months (the 6-month cohort). 


\section{LIMITATIONS}

There are a number of limitations to our results. First, outcomes of VA nursing home residents may be different than for community nursing home residents. The most obvious difference is gender. In VA nursing homes, $96.1 \%$ of residents are men compared to $28.4 \%$ of residents in community nursing homes (Mehr, Fries, \& Williams, 1993). Because of the very small numbers of women, we did not consider gender separately in our analysis. However, we restricted our study population to those at least 65 years of age. Older VA nursing home residents are more similar to the community nursing home population than all VA nursing home residents (Mehr et al., 1993). Nonetheless, our findings should be extrapolated with caution to community nursing home residents.

Second, we have very limited data on social resources. Other than current marital status, our administratively derived data have no information on social supports or community resources. As previously noted, almost all veterans were low income. The high proportion of low-income residents in the VA nursing home system is not without parallel. A reported 50\% of residents received Medicaid in the 1985 National Nursing Home Survey (Hing, 1989).

Third, as with any study using administrative data, potential data quality problems exist. Completeness and accuracy of diagnosis coding, reliability of assessment information, and accuracy in recording transitions are all potential problems. We used all coded diagnoses rather than just the diagnosis responsible for the stay to reduce the probability of missing important diagnoses. Although we have no specific information on reliability of VA assessments, similar instruments have been found reliable in several states. We did find recording of admission source and discharge destination to be unreliable. We dealt with this by avoiding almost entirely the use of these codes. We identified transitions when individuals had records sequentially from two different settings. The one exception was discharge out of care under VA auspices where we noted if they went to another long-term care setting. 


\section{CONCLUSIONS}

Despite rich data resources, we found that models for predicting death and community discharge were only modestly predictive after the initial period of nursing home stay. Further, with a few exceptions-notably age, ADL status, and identification as terminally illpredictors varied considerably between death and community discharge and with differing length of stay. Our findings reinforce the difficulty in using resident-care transitions in any outcome-based reimbursement or quality improvement system. Despite the immense increase in assessment information now becoming available with the implementation of the Minimum Data Set for Resident Assessment and Care Planning (MDS) (Morris et al., 1990), we are pessimistic that substantially improved predictive models can be developed for the overall population of nursing home residents. There may be specific subgroups for whom it is possible to better estimate expected trajectory toward death or community discharge. However, at least in the short term, quality improvement in nursing homes will be better served by using commonly accepted care outcomes, such as ADL status, catheter use, and the presence or absence of decubitus ulcers (Institute of Medicine, 1986; Kane, 1995).

\section{REFERENCES}

Engle, V.F., \& Graney, M. J. (1993). Predicting outcomes of nursing home residents: Death and discharge home. Journal of Gerontology, 48, S269-S275.

Greene, V. L., \& Ondrich, J. I. (1990). Risk factors for nursing home admissions and exits: A discrete-time hazard function approach. Joumals of Gerontology, 45, S250-S258.

Hing, E. (1989). Nursing home utilization by current residents: United States, 1985 (DHHS Pub. No. PHS 89-1763). Washington, DC: Government Printing Office.

Hing, E., Sekscenski, E., \& Strahan, G. (1989). The national nursing home survey: 1985 summary for the United States (DHHS Pub. No. PHS 89-1758, pp. 1-249). Washington, DC: Government Printing Office.

Hosmer, D. W., Jr., \& Lemeshow, S. (1989). Applied logistic regression. New York: Wiley.

Institute of Medicine, Committee on Nursing Home Regulation. (1986). Improving the quality of care in nursing homes. Washington, DC: National Academy Press.

Kane, R. L. (1990). Rethinking long-term care. Journal of the American Geriatrics Society, 38, 704-709. 
Kane, R. L. (1995). Improving the quality of long-term care. Journal of the American Medical Association, 273, 1376-1380.

Kane, R. L., Bell, R., Riegler, S., Wilson, A., \& Keeler, E. (1983). Predicting the outcomes of nursing home patients. Gerontologist, 23, 200-206.

Keeler, E. B., Kane, R. L., \& Solomon, D. H. (1981). Short- and long-term residents of nursing homes. Medical Care, 19, 363-370.

Kiel, D. P., Eichorn, A., Intrator, O., Silliman, R. A., \& Mor, V. (1994). The outcomes of patients newly admitted to nursing homes after hip fracture. American Journal of Public Health, 84, 1281-1286.

Lewis, M. A., Cretin, S., \& Kane, R. L. (1985). The natural history of nursing home patients. Gerontologist, 25, 382-388.

Lewis, M. A., Kane, R. L., Cretin, S., \& Clark, V. (1985). The immediate and subsequent outcomes of nursing home care. American Journal of Public Health, 75, 758-762.

Lewis, M. A., Leake, B., Clark, V., \& Leal-Sotelo, M. (1989). Case mix and outcomes of nursing home patients: The importance of prior nursing home care and admission from home versus hospital. Medical Care, 27, 376-385.

Mehr, D. R., Fries, B.E., \& Williams, B. C. (1993). How different are VA nursing home residents? Joumal of the American Geriatrics Society, 41, 1095-1101.

Morris, J. N., Hawes, C., Fries, B. E., Phillips, C. D., Mor, V., Katz, S., Murphy, K., Drugovich, M. L., \& Friedlob, A. S. (1990). Designing the national resident assessment instrument for nursing homes. Gerontologist, 30, 293-307.

Murtaugh, C. M., Cooney, L. M., DerSimonian, R. R., Smits, H. L., \& Fetter, R. B. (1988). Nursing home reimbursement and the allocation of rehabilitation therapy resources. Health Services Research, 23, 467-493.

Narain, P., Rubenstein, L. Z., Wieland, G. D., Rosbrook, B., Strome, L. S., Pietruszka, F., \& Morley, J. E. (1988). Predictors of immediate and 6-month outcomes in hospitalized elderly patients: The importance of functional status. Joumal of the American Geriatrics Society, 36, 775-783.

Retsinas, J., \& Garrity, P. (1986). Going home: Analysis of nursing home discharges. Gerontologist, 26, 431-436.

The SAS system for Windows (Release 6.08) [Computer software]. (1992). Cary, NC: SAS Institute, Inc.

Schneider, D. P., Fries, B. E., Foley, W. J., Desmond, M., \& Gormley, W. J. (1988). Case mix for nursing home payment: Resource utilization groups, version II. Health Care Financing Review, Spec No (Annual suppl.), 39-52.

Spence, D. A., \& Wiener, J. M. (1990). Nursing home length of stay patterns: Results from the 1985 National Nursing Home Survey. Gerontologist, 30, 16-20.

Wayne, S. J., Rhyne, R. L., Thompson, R. E., \& Davis, M. (1991). Sampling issues in nursing home research. Joumal of the American Geriatrics Society, 39, 308-311.

Weissert, W. G., \& Scanlon, W. J. (1985). Determinants of nursing home discharge status. Medical Care, 23, 333-343.

Williams, B. C., Demitrack, L. B., \& Fries, B. E. (1992). The accuracy of the National Death Index when personal identifiers other than Social Security number are used. American Joumal of Public Health, 82, 1145-1147.

Williams, B. C., Fries, B. E., \& Mehr, D. R. (1993). Length of stay in VA nursing homes: Comparative characteristics of brief-, medium-, and long-stay residents. Joumal of Aging and Health, 5, 208-228. 
Williams, B. C., Fries, B. E., \& Mehr, D. R. (1996). Patterns and determinants of health services use and mortality after VA nursing home care. Joumal of Aging and Health, 8, 280-301.

Williams, B. C., Mehr, D. R., \& Fries, B. E. (1994). Use of administrative records to describe longitudinal patterns of veterans' health services use. Jourmal of the American Medical Informatics Association, 1, 325-327. 